\title{
Strategies proposed by the Word Health Organization for the combat of smoking
}

\author{
Serviço de Prevenção do Instituto do Coração do Hospital \\ das Clínicas da Faculdade de Medicina da USP
}

\section{World Scenario:}

Smoking is presently considered the greatest avoidable risk factor in the prevention of diseases. It is not a natural factor like a virus or a bacteria or even a factor brought about by a natural catastrophe; it is produced by man. Despite all the evidence of the health hazards caused by smoking, its existence is perpetuated because of strong economic interests.

The prevalence of smoking is very high in the world. It reaches $50 \%$ of the adult male population. This percentage is frequently seen in developing countries, but it is also very high in developed countries, where it reaches $41 \%$. In the female population, the situation is reversed. Approximately only $8 \%$ of the women smoke in developing country, whereas $21 \%$ of them do in developed countries.

Table I shows the prevalence of smoking in the general population and among physicians in certain countries. Even in countries considered developed, the prevalence of smoking is high in both the general population as well as among physicians. This clearly shows the wide social acceptance of tobacco as a drug. This acceptance is proportional to the degree of advertising campaigns favorable to cigarette smoking.

Nowadays, due to measures adopted in developed countries aimed at restraining cigarette advertising, tobacco company advertising interest has shifted to Latin America, Eastern Europe and Asia (China). Whereas tobacco consumption declined 13\% from 1985 to 1992 in developed countries, it increased 20\% in China.This means that for each cigarette not lighted in developed countries, three were lighted in China.

Annual cigarette consumption in the 1980s declined 2,800 cigarettes/person/year to 2,400 cigarettes/person/year in the developed countries, whereas in developing countries it jumped from 1,150 cigarettes/person/year to 1,400 cigarettes/person/year in the same time period.

\section{Reasons for the adoption of measures restricting} tobacco:

The WHO estimates that there are presently 1.1 billion smokers. When one considers that $50 \%$ of them die as a result of smoking, it is expected that 500 million people die because of tobacco. Half of these deaths are middle age individuals, which means a life expectancy reduction of 20 to 25 years. Presently, cigarette smoking is responsible for $20 \%$ of all deaths. It kills 3 million people every year worldwide. If the trend of increasing cigarette consumption persists in the developing countries, 7 million people per year are expected to die from smoking in the next two decades.

\section{B. Economic features}

These early deaths as well as the high morbidity associated with cigarette smoking result in higher costs for the public health system of any country, be it rich or poor.

According to H. Barnum, a World Bank economist, tobacco is considered the greatest waste of financial resources in the world(1). In his study, Barnum determined that for an increase in 100 tons of tobacco consumed per year, an additional 650 deaths per year would mean generating losses in the excess of U.S. \$27.2 million.

U.S. $\$ 200$ billion are lost worldwide on an annual basis as a result of smoking-related morbidity and mortality.

Manufacturing a cigarette is good business for tobacco companies. One kilogram of tobacco is bought from the tobacco grower for U.S. $\$ 1.65$. After the drying process, the same kilogram will cost U.S. $\$ 3.00$ and it will be enough to make 1,200 cigarettes. Considering that manufacturing is entirely automated and that the product has a long life, it is clear that manufacturing costs are very low. Additionally, there is 
always a guaranteed consumer due to the high degree of addiction tobacco creates.

C. Environmental features:

The tobacco leaf-drying process requires, wood. In the drying process, one kilogram of tobacco leaves requires 100 kilograms of wood. Despite the requirement that tobacco companies reforest areas where the wood is extracted from, the rate of tree growth and tree replacement is considerably lower than extraction and demand.

The awareness that environmental exposure to cigarette smoke leads to illness in non-smoking subjects has given a new spark to the anti-tobacco campaign. This new reality changed the social acceptability regarding cigarette smoking. Several companies had to adopt smoking prohibition policies in the work place because of numerous lawsuits initiated against them since 1976. In this year, a Bell Telephone Company non-smoking phone operator won a lawsuit giving her the right to work in a smoke-free place. She had severe allergic reactions when exposed to cigarette smoke.Today nearly $60 \%$ of companies in the United States have anti-smoking rules in the work place. In the 1980 s, only $16 \%$ of companies adopted these measures. 